Provisional figures show that in 1989 the number of live births stated at conception to have been unplanned was $27 \%$, but it dropped to $15 \%$ after delivery, which reflects the natural adaptation and amnesia that occurs in these circumstances. If those pregnancies that end in a miscarriage or termination are included, the original unplanned $40 \%$ contrasts with the $15 \%$ figure recorded after delivery.

Although Alton may not compare with the 20 areas of England and Wales chosen by Ms Fleissig, they suggest that our overall figure for unplanned pregnancies was about twice what would have been recorded by restricting any such inquiry to those women who had had a baby some months earlier.

Ms Fleissig has exposed the deteriorating effectiveness of our current policies on young users of the pill and that there is an urgent need for improved care. These unwanted pregnancies create most of the abortions carried out every year; many produce unwanted children. As the problems seem to follow failure of the contraceptive pill, do we now need to increase the strength of oral contraceptives used by young women?

Alton Health Centre, Alton,

C B EVERETT

Hampshire GU34 2QX

1 Fleissig A. Unintended pregnancies and the use of contraception :

changes from 1984 to 1989. BMJ 1990;302:147. (19 January.)

\section{Early pregnancy assessment}

SIR, - In their recent paper on the management of women referred to an early pregnancy assessment unit Drs M A Bigrigg and M D Read did not say whether ultrasound scans were done using the transabdominal or transvaginal route. 'Based on the number of equivocal scan results which they reported $(11 \%)$, it is likely that the transabdominal route was used.

We have done a pilot study to assess the impact of transvaginal ultrasound scanning of emergency gynaecological cases during the first three months of its use in our department. The admitting doctor made a tentative clinical diagnosis on the basis of the patient's history and physical findings. Transvaginal ultrasonography was done by medical staff; the facility was available 24 hours a day, seven days a week. All scans were done within 12 hours of admission, but most were done within a few hours of the patient's arrival in hospital. A transvaginal Endo V probe (Siemens) with a Sonoline SL-2 ultrasound system was used.

During the study period transvaginal scans were done on 87 patients referred with bleeding or pain in early pregnancy. In 72 a clinical diagnosis of threatened abortion had been made, and in 15 there was clinical suspicion of ectopic pregnancy. After a single scan a definite and conclusive diagnosis was made in 67 of the 72 patients with a clinical diagnosis of threatened abortion (viable intrauterine pregnancy, 27; retained products of conception, 21 ; missed abortion, 8 ; blighted ovum, 8 ; complete abortion, 4$)$. In the remaining four patients the ultrasound findings suggested ectopic pregnancy. In three of these a diagnosis of tubal pregnancy was confirmed at laparoscopy, and in one a diagnosis of complete abortion was subsequently made.

A correct and definite diagnosis was made after a single scan in seven of the 15 patients in whom there was clinical suspicion of ectopic pregnancy. At ultrasonography a live intrauterine fetus was seen in one case, retained products of conception in four, and a viable intrauterine pregnancy in two. In eight a definite diagnosis could not be made at ultrasonography, but the findings suggested ectopic pregnancy, which was confirmed at laparoscopy.

Transvaginal ultrasonography has many advantages over transabdominal ultrasonography, particularly in the management of patients with bleeding and pain in early pregnancy. The quality of the image obtained by using vaginal ultrasonography is vastly superior to that achieved by using abdominal scanning. A clear gestational sac can be detected in all patients at 4.5 weeks after the last menstrual period, and fetal heart beats are detected by the end of the fifth week. ${ }^{2}$ In most cases the superior quality of the image achieved with the vaginal probe allows a definite diagnosis of nonviability to be made with confidence after a single scan at an earlier gestational age, obviating the need to repeat a scan at a later date. In addition, transvaginal ultrasonography is superior to transabdominal ultrasonography in detecting ectopic pregnancy. ${ }^{3}$

During our pilot study we found that one of the main advantages of transvaginal ultrasonography was the saving in time taken to make a diagnosis as emergency cases could be scanned on admission without waiting for the bladder to fill. The patients were starved from the time of admission so that there was no delay after scanning for those patients requiring surgery. The potential savings in hospital resources, particularly in terms of bed occupancy, that can result from the introduction of this technique are considerable.

PAUL BYRNE MICHAEL JOHN RICHARD KENNEDY KRYS CIETAK

Department of Obstetrics and Gynaecology, Walsgrave Hospital, Coventry CV2 2DX

1 Bigrigg MA, Read MD. Management of women referred to early pregnancy assessment unit: care and cost effectiveness. $B M$ 1991;302:577-9. (9 March.)

2 Timor-Tritsch E, Rottem S, Thaler I. Review of transvagina ultrasonogrpahy: a description with clinical application Ultrasound Quarterly 1988;6:1-34.

3 Shapiro BS, Cullen M, Taylor KJW, DeCherney AH. Trans vaginal ultrasonography for the diagnosis of ectopic pregnancy. Fertil Steril 1988;50:425-9.

\section{Anaesthetists against incompetence}

SIR, - I must correct a historical point in Dr Richard Smith's article on the college's scheme for competence. ${ }^{\prime}$ The sick doctor scheme, the first in the United Kingdom, was introduced by the Association of Anaesthetists of Great Britain and Ireland. This occurred promptly through the coincidence that the president, Dr C F Scurr, had been a member of the Alment committee, which produced an important report, and I, as secretary, had close contact in Cardiff with Professor K Rawnsley, then president of the Royal College of Psychiatrists. Vital for acceptance, as for the present scheme, was the confidence shown by anaesthetists in their organisations and, more importantly, in themselves.

College of Anaesthetists

MICHAEL ROSEN

London WC2A 3PN

1 Smith R. Anaesthestists against incompetence. BMF 1991;302 371. (16 February.)

SIR,-Dr Richard Smith reports that the College of Anaesthetists has decided that doctors should be disciplined if they have a "high incidence of complications as evidenced by audit, or fail to comply with generally accepted practice." This represents a great threat to clinical freedom. When medical audit was introduced promises were made that it would not be used against individual doctors; education and persuasion were the keys to improvement. Now audit is to be a stick to force doctors into complying with the prejudices of the majority.
For hundreds of years medical progress was blocked because doctors were compelled to comply with "generally accepted practice," as had been taught by Galen. Happily those days passed and doctors such as Withering, Lister, Semmelweis, Pinel, and Morton, none of whom complied with "generally accepted practice," were able to make their great contributions to medical progress.

The present proposals will stifle medical innovation and flexibility as original thinkers are compelled to conform. No one likes his or her cherished nostrums to be challenged, and hence innovators automatically generate hostility. Assessors, before condemning a colleague, should exercise the same scientific and statistical standards as they would before accepting any other medical evidence. Anything less is glorified witch hunting. In the mean time perhaps doctors should consider abstaining from any audit lest they create rods for their own backs.

Wiltshire SP5 3HJ

P J TOMLIN

I Smith R. Anaesthetists against incompetence. BMf 1991;302: 371. (16 February.)

\section{Hormone replacement therapy and ovarian cancer}

SIR,-Despite concern that hormone replacement therapy may hasten the spread of disease in women with ovarian cancer Dr R A Eeles and colleagues report that the survival of women who received such therapy was similar to, or possibly better than, that of women who did not receive it. Interpretating their data, however, is not straightforward as they show clearly that hormone replacement therapy was given preferentially to women with a good prognosis: young women and women with early stage disease and well differentiated tumours.

The prognostic factors analysed are relatively crude predictors of survival, and statistical adjustment for them does not ensure that the groups compared are similar in other respects. Unmeasured features, such as the woman's progress after ovarian cancer was intially diagnosed, are likely to have influenced the decision to give hormones and would also have a profound effect on survival. Differences in prognosis between the treated and untreated groups that are not explained by the factors analysed in their paper might even be so great that they mask a detrimental effect of the therapy.

We believe that the influence of hormone replacement therapy on survival cannot be reliably judged from this study, and we agree with the authors that the only way to resolve this question is with a randomised trial.

VALERIE BERAL SARAH DARBY

Imperial Cancer Research Fund

Radcliffe Infirmary

Oxford OX2 6HE

Imperial Cancer Research Fund,

London WC2A 3PX

1 Eeles RA, Tan S, Wiltshaw E, et al. Hormone replacement therapy and survival after surgery for ovarian cancer. $B M Y$ therapy and survival after surgery

\section{Reporting of fine needle aspiration}

SIR, - As a histopathologist who takes and immediately reports on fine needle aspirates of the breast (and other sites) in outpatients I was delighted to see the article by $\mathrm{Mr} \mathrm{J}$ Michael Dixon advocating this practice.' I concur with his comments that this facility is sensitive and lessens patients' anxiety 